\title{
USING PROXIMITY TECHNOLOGY FOR LOCALIZATION IN WIRELESS SENSOR NETWORKS
}

The localization in wireless sensor networks is very important for function of the network (routing...). The objective of the localization process is to determine the position of device (sensor, node) from the information acquired from the reference devices. These devices of course know their location. The aim of the paper is to give a survey of two proximity based location techniques performance. The results of basic proximity based localization techniques are compared with modified (enhanced) proximity technique. It does not belong to the most accurate techniques, but on the other hand, it is a low cost alternative to more expensive techniques.

\section{Introduction}

The physical world will consist of large networks of wireless sensor nodes. Particularly, applications like environmental monitoring of water and soil require that these nodes be very small, light and unobtrusive. A wireless sensor network consists of a possibly large number of wireless devices able to take environmental measurements. Typical examples are temperature, light, sound, and humidity. These sensor readings are transmitted over a wireless channel to a running application that makes decisions based on these sensor readings. Many applications have been proposed for wireless sensor networks, and many of these applications have specific quality of service (QoS) requirements that offer additional challenges to the application designer. Several applications have been envisioned for wireless sensor networks [1]. These range in scope from military applications to environment monitoring to biomedical applications. This paper deals with localization by using proximity technology.

In this paper, we review localization techniques and evaluate the effectiveness of a very simple proximity (connectivity-metric) method for localization in all environments that make use of the inherent radio-frequency (RF) communications capabilities of these devices.

\section{Localization in Wireless Networks}

The concept of location is not limited to the geographic representation of physical location with sets of coordinates (latitude, longitude, and altitude). It is also applicable to symbolic location in a nongeographic sense such as location in time or in a virtual information space such as a data structure or the graph of a network.

Common to all notions of location is the concept that the individual locations are all relative to each other, meaning that they depend on a predefined frame of reference. This leads to a differentiation of the relative and absolute positioning cases [2].

When talking about physical location in the traditional way, points are usually viewed as three-dimensional coordinates $[x, y$, $z$ ] in a Cartesian reference coordinate system. Many other transformations to other coordinate systems like polar coordinates are equivalent.

Usually, $[x, y, z]$ coordinates by themselves are not meaningful for context-aware system services and other information needs to be associated with these position fixes. In these cases, it is important to introduce the fourth dimension - time, to be able to specify where and when a certain event took place resulting in sets of $[x, y, z, t]$ for each position fix. This four-dimensional fix can then be used to put subsequent events into a context frame.

When position information is used in reference to a geographic map or a global time reference, context information can be extended. The position of node can be defined as absolute or relative position. An absolute position is given in respect to an inertial system and a reference point in this inertial system (see Fig. 1 a). On the other hand, a relative position can only be given in respect to other points resolving the distances and the geometric configuration, e.g., the topology (see Fig. $1 \mathrm{~b}$ ). It means that absolute positioning data are used with respect to the absolute position of other nodes or to a reference position or a map. Relative positioning can discriminate topology information only in a node's local reference system.

Localization techniques that use positioning generally consist of three components [2]:

- Identification and data exchange.

- Measurement and data acquisition.

- Computation to derive location.

\footnotetext{
* Peter Brida, Martin Matula, Jan Duha

Department of Telecommunications, Faculty of Electrical Engineering, University of Žilina, Slovakia E-mail: peter.brida@fel.uniza.sk,
} 


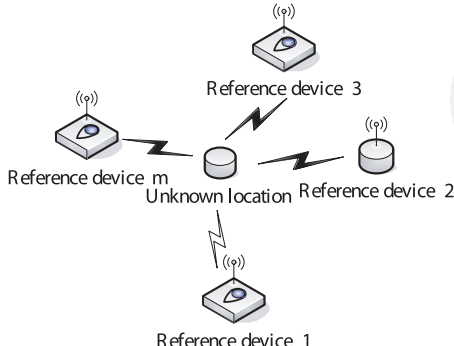

a)

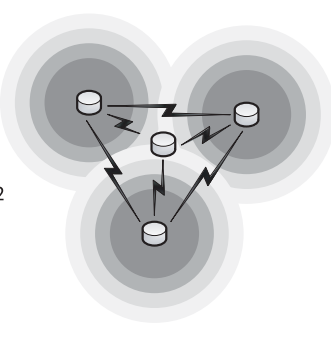

b)
Fig. 1 a) Absolute positioning b) Relative positioning

The various approaches divide these tasks differently across their system components.

The mechanisms used for estimating location are possible to divide into two categories: range-based and range-free [3]. The range-based mechanisms are based on the indirect measurements of distance or angle between sensors. Examples of the mechanism are the following methods: received signal strength indicator (RSSI), time of arrival (ToA), time difference of arrival (TDoA), carrier phase and code measurements, ultra wide-band (UWB), ultrasound, and even visible light pulses or the angle of arrival (AoA) of a radio signal. The important thing to note is that these mentioned measurements always have errors and individual measurements are not independent of each other and are strongly influenced by the surrounding environment and the used transmission system.

The devices in some systems (e.g., wireless sensor networks) have the hardware limitations. To overcome the limitations of the range-based positioning methods, many range free solutions have been proposed. These solutions estimate the location of mobile devices (sensors, nodes or mobile stations) either by exploiting the radio connectivity information among neighboring mobile devices, or by exploiting the sensing capabilities of the mobile devices. Therefore the range-free positioning solutions are cost-effective alternative to more expensive range based approaches.

Due to the distinct characteristics of these two approaches, we categorize the range-free positioning methods into: reference device based methods and reference device free methods. For the first method, the presence of devices, that have knowledge about their location, is assumed in the network. And the second method requires no special sensor nodes for positioning.

\subsection{Location in Wireless Sensor Networks}

Wireless Sensor Networks (WSNs) are the special part of wireless ad hoc networks. The localization in WSNs is a little bit different in comparison with localization in traditional wireless networks (e.g. cellular networks).

WSN is usually characterized by subjects such as vast number of devices, high overall node mobility, considerable power and resource consumption at the nodes, and moderate network sizes,
WSN have brought about quite a change in the traditional, connection-oriented, infrastructure-dominated telecommunications world [2]. Unpredictable dynamics due to failures and changes in nodes and the environment, as well as deployment in uncontrolled areas with high dynamics and possibly hostile to radio signal propagation, require adaptable networking mechanisms. Other characteristics of typical WSN nodes are the limited resources available on these low power embedded systems, especially the limited transmission range and low duty cycle operation of the radio transceivers. Nodes can be reactive and are able to wake up on demand [2]. Targeted for a very long lifespan, integrated into all kinds of everyday objects and building materials, deployed once, and in many cases never collected again or decommissioned, the vast majority of nodes will form a quasistatic, multihop network topology (see Fig. 2).

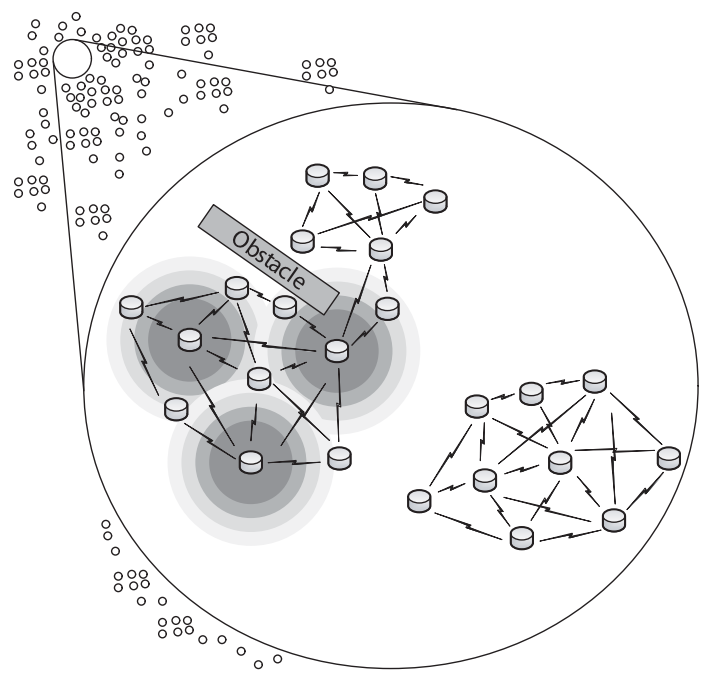

Fig. 2 Topology of wireless sensor network

The problem of localization, i.e., determination where a given node is physically located in a network is a challenging one, and yet extremely crucial for many of these applications. Practical considerations such as the small size, form factor, cost and power constraints of nodes preclude the reliance on Global Positioning System (GPS) on all nodes in these networks.

GPS may solve the problem of localization in outdoor environments for nodes. However, for large networks of very small, inexpensive, and low-power devices, practical considerations such as size, form factor, cost, and power constraints of the nodes preclude the use of GPS on all nodes. The GPS-less system [1] addresses the problem of localization for such devices, with the following design goals:

- RF based: they focus on small nodes that have some kind of short-range RF transceiver. The primary goal is to leverage this radio for localization, thereby eliminating the cost, power, and size requirements of a GPS receiver.

- Receiver based: in order to scale well to a large distributed network, the responsibility for localization must lie with the 
receiver node that needs to be localized, and not with the reference points.

- Ad hoc: in order to ease deployment, a solution that does not require preplanning or extensive infrastructure is desired.

- Responsiveness: we need to be able to localize within a fairly low response time.

- Low Energy: small, untethered nodes have modest processing capabilities, and limited energy resources. If a device uses all of its energy localizing itself, it will have none left to perform its task. Therefore, we want to minimize computation.

- Adaptive Fidelity: in addition, we want the accuracy of our localization algorithms to be adaptive to the granularity of available reference points.

\subsubsection{Proximity Based Localization}

Localization using proximity measurements is popular, when low cost takes precedence in priority over accuracy. Since, messages necessarily pass between neighbors, there is no additional bandwidth required to proximity. Proximity measurements simply report whether or not two devices are 'connected' or 'in-range'. However, the term 'in-range' may mislead readers to believe that proximity is purely a function of geometry - whether or not two devices are separated by less than a particular distance. In fact, proximity is determined by whether or not a receiver can demodulate and decode a packet sent by a transmitter. Given the received signal and noise powers, the successful reception of a packet is a random variable. Yet, proximity carries considerable information regarding sensor location in a binary variable. The proximity has been used by numerous researchers for localization in ad hoc networks and wireless sensor networks $[4,5,6,7]$.

A fixed number of reference points in the network with overlapping regions of coverage transmit periodic beacon signals. Nodes use a simple connectivity metric that is more robust to environmental vagaries, to infer proximity to a given subset of these reference points. Devices localize themselves to the centroid of their proximate reference points. The accuracy of localization is then dependent on the separation distance between two adjacent reference points and the transmission range of these reference points.

\section{System and Measurement Models}

WSNs are made up of peer-to-peer links between devices (nodes). Pair-wise measurements can be made from any of these links, but only a small fraction of devices have coordinate knowledge. Thus, the measurements are made primarily between pairs of devices of which neither has known coordinates. The device with known coordinates is called a reference device. Otherwise, it is referred to as blindfolded device, since it cannot see their location. Specifically, consider a network of $m$ reference and $n$ blindfolded devices. The relative location problem corresponds to the estimation of blindfolded device coordinates. For simplicity we consider the location in 2D plane. Let $\left[X_{i}, Y_{i}\right]^{T} i=1,2, \ldots, m$ are coordinates of reference devices and $\left[X_{j}, Y_{j}\right]^{T} j=1,2, \ldots, n$ are coordinates of blindfolded devices. Pair-wise measurements $\left[R S S_{i, j}\right]$, where $R S S_{i, j}$ is a measurement between devices $i$ and $j . R S S_{i, j}$ is received signal strength in device $j$ from the device $i$.

\subsection{Channel Model}

We consider a fading channel in all measurements in this paper. The propagation path between two devices is shown in fig. 3 , where $R S S$ is received signal strength, $T_{x}$ is transmitted signal strength. The influence signal attenuation is defined by: $L_{L S}$ is signal degradation caused by large-scale propagation, $L_{M S}$ is signal degradation caused by medium-scale propagation and finally $L_{S S}$ is signal degradation caused by small-scale propagation. All these parameters are in $[\mathrm{dBm}]$. Parameters $L_{M S}$ and $L_{S S}$ have the normal distribution, but their impact on the proximity based localization is negligible, because most of devices are static and these parameters are primarily changed during motion of device. Therefore, we take into consideration idealized radio model only $L_{L S}$. We chose this model because it is mathematically simple and easy. In this section, this model is presented. We make two assumptions in the model: the perfect spherical radio propagation and identical transmission range (power) for all devices.

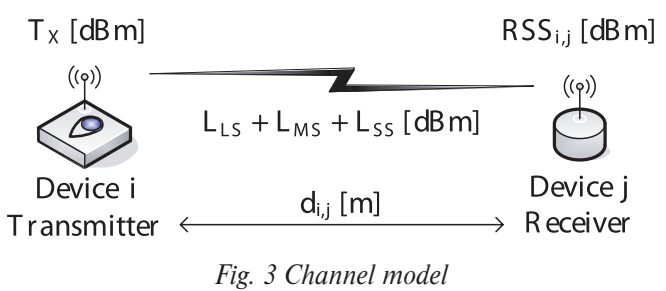

$R S S_{i, j}$ is the measured received signal strength at device $j$ transmitted by device $i$ (in $\mathrm{dBm}$ ) is Gaussian

$$
\operatorname{RSS}_{i, j}(d B m)=P_{0}(d B m)-10 \cdot n_{p} \cdot \log \left(d_{i, j} / d_{0}\right),
$$

where $R S S_{i, j}$ is the mean power in $\mathrm{dBm}, P_{0}$ is the received signal strength at the reference distance $d_{0}$. Typically $d_{0}=1$ meter, and $P_{0}$ is calculated by the free space path loss formula [2]. The path loss exponent $n_{p}$ is a function of the environment.

\subsection{Proximity Measurements}

The proximity measurement $S$ is determined based on the measured signal. $S_{i, j}$ is obtained from $R S S_{i, j}$ and it is equal to 1 if devices $i$ and $j$ are in range, and it is 0 if not. It is necessary to clearly define transition from status "in range" to "out of range". Therefore, we define a threshold. If the received signal strength $\left(R S S_{i, j}\right)$ at $j$ device transmitted by $i$ device is higher as defined threshold then $i$ devices is assumed to be in range of the $j$ device. Thus,

$$
S_{i, j}=\left\{\begin{array}{ll}
1, & R S S_{i, j} \geq R S S_{T} \\
0, & R S S_{i, j}<R S S_{T}
\end{array} .\right.
$$




\section{Simulation Environment}

The presented numerical simulations compare the performance of various solutions of proximity localization. Simulations are done for one propagation environment. The all devices are situated in square $10 \mathrm{~m} \times 10 \mathrm{~m}$. The reference devices are situated randomly (according to Gaussian distribution $N(0,1)$ ) in mentioned area. Five hundred trials are performed. In each trial, the positions of reference devices and blindfolded device are generated at first. In the next step, RSS are calculated between each reference devices and blindfolded device based on the equation (1). Here, we use the parameters $n_{p}=2$ (free space). Then, the devices are rejected which do not fulfill the threshold condition. The location coordinates of blindfolded device are calculated based on the remaining reference devices. The location coordinates can be calculated based on the following techniques (our working titles):

- Common proximity - the estimated location is determined on the basis of the closest device location, i.e. the blindfolded device has same location coordinates as the closest device.

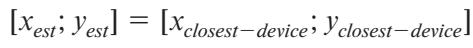

- Centroid proximity - the position of blindfolded device is calculated as mean value of coordinates $N$ of the closest reference devices. It is defined by the centroid of these reference devices (see equation (4)).

$$
\left[x_{\text {est }} ; y_{\text {est }}\right]=\left[\frac{1}{N} \sum_{i=1}^{N} x_{i} ; \frac{1}{N} \sum_{i=1}^{N} y_{i}\right]
$$

The accuracy of device positioning is compared by means of RMSE (Root Mean Square Error)

$$
R M S E=\sqrt{\left(x_{r}-x_{e s t}\right)^{2}+\left(y_{r}-y_{e s t}\right)^{2}}[\mathrm{~m}],
$$

where $\left[x_{r} ; y_{r}\right]$ are coordinates of the real (precise) position and $\left[x_{\text {est }} ; y_{\text {est }}\right]$ are coordinates of estimated position on the basis of the selected technique.

\section{Simulation Results}

In this section, we discuss obtained results by means of techniques described in the previous part. The results are simulated to evaluate the performance of the proximity localization in WSNs. The simulations are realized for two cases:

- Optimization of the number reference devices used for location estimation in centroid proximity.

- Influence of the number of all reference devices used in simulation of both proximity techniques.

In the first case was surveyed an influence of $N$ number of reference devices used for the location estimation in centroid proximity. In the following figures are shown the results for two different numbers of reference devices used in simulation. The overall number of reference devices was 10 and 100 . The experiments were realized for more values of $m$ with similar results.
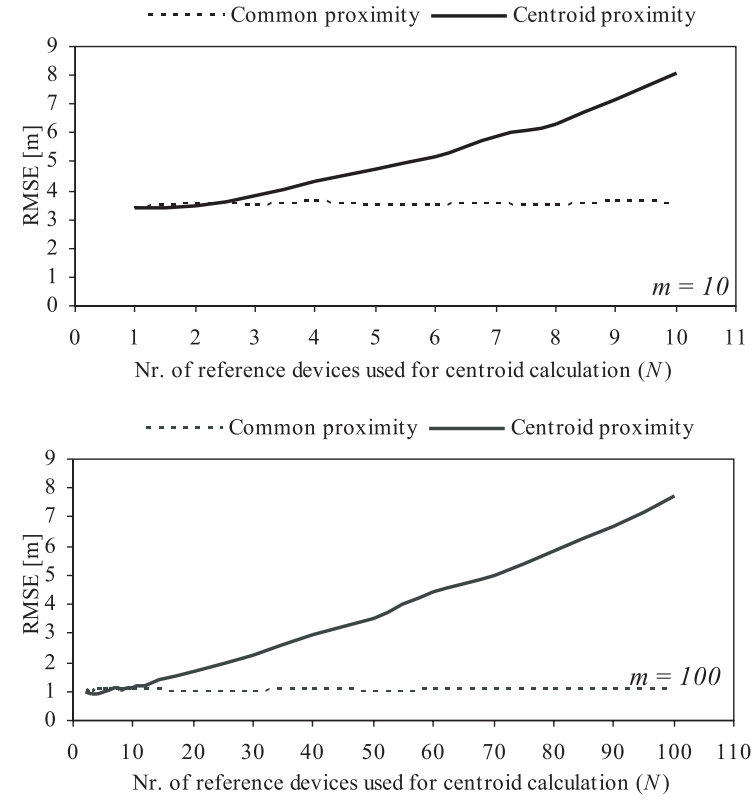

Fig. 4 RMSE [m] versus number of reference devices used for centroid proximity calculation

Fig. 4 depicts RMSE localization error properties in the cases for two different numbers of all devices used in simulation and the dependency of localization error on $N$ (the number of reference devices used for calculation of centroid proximity location estimation). Naturally, the change of $N$ doesn't impact on accuracy of common proximity (dash line). The number of reference devices used for estimation calculation plays an important role in localization accuracy. The minimal error is interesting. The results confirm the fact that centroid proximity achieves the smallest localization error in comparison of common proximity based technique. The difference of the minimal localization error is the most important factor of an objective comparison for the observed techniques. In both observed cases of $m(m=10$ and 100), the minimal RMSE was obtained for case $N=2$. Therefore in next simulation will be used only two reference devices for calculation of device coordinates in centroid proximity. In general, we can say that the accuracy

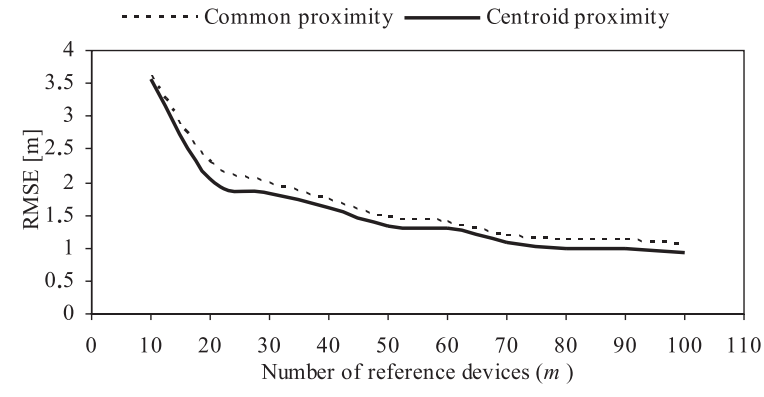

Fig. 5 RMSE $[\mathrm{m}]$ versus number of reference devices for both proximity techniques 
decreases as the number of reference devices used for centroid proximity calculation (except $N=2$ ) increases. Therefore, centroid proximity technique is suitable to use for estimation of location.

The following figure shows the performance comparison of common and centroid proximity for the same number of reference devices used for location estimation.

Fig. 5 shows the dependence of the RMSE on the number of reference devices $m$. For calculation of centroid proximity were used the two closest reference devices. On a basis of the obtained results we conclude that the centroid proximity technique achieves

RMSE and STD [m] versus number of reference

Table 1 devices for both proximity techniques

\begin{tabular}{|c|c|c|c|c|}
\hline \multirow{2}{*}{$\begin{array}{c}\text { Nr. of } \\
\text { reference } \\
\text { devices }\end{array}$} & \multicolumn{4}{|c|}{ Proximity } \\
\cline { 2 - 5 } & RMSE [m] & STD [m] & RMSE [m] & STD [m] \\
\hline 10 & 3.6372 & 1.9538 & 3.5644 & 2.0515 \\
\hline 20 & 2.3049 & 1.2067 & 2.0434 & 1.3152 \\
\hline 30 & 1.9693 & 1.0003 & 1.8265 & 1.1258 \\
\hline 40 & 1.7319 & 0.951 & 1.618 & 0.9931 \\
\hline 50 & 1.4721 & 0.8022 & 1.3357 & 0.7734 \\
\hline 60 & 1.3863 & 0.7407 & 1.2943 & 0.7565 \\
\hline 70 & 1.1892 & 0.6859 & 1.0708 & 0.7134 \\
\hline 80 & 1.1061 & 0.5982 & 0.9853 & 0.6007 \\
\hline 90 & 1.1182 & 0.6332 & 1.0008 & 0.6507 \\
\hline 100 & 1.0644 & 0.5552 & 0.9246 & 0.5572 \\
\hline
\end{tabular}

more accurate results comparing to centroid and common proximity. The RMSE is an exponential function of the number of reference devices $m$. Ascending value of the reference devices number means increasing of the RMSE.

The results (statistical characteristics) are compared and numerically expressed in Table 1 . The RMSE is an average value of all 500 trials for one scenario and the STD is a standard deviation also for the same scenario.

\section{Conclusions}

We discussed two techniques for the location determination based on the proximity technology for wireless sensor networks. We compare the common proximity with centroid proximity technique. According to the results, the performance of the centroid proximity technique is better in comparison to the common proximity when two reference nodes are used for calculation of position coordinates. Hence, it is possible to recommend using the centroid proximity with application of two reference devices for positioning. The common proximity achieved the worst results, but this variant does not need any calculation capacity for the localization procedure. The method is not accurate in comparison with the sophisticated localization methods, but it is sufficient for the less demanding applications in huge WSNs.

\section{Acknowledgments:}

This paper is based on the work supported by the grant VEGA 1/4065/07 of Scientific Grant Agency of the Slovak Republic.

\section{References:}

[1] STOJMENOVIC, I.: Handbook of Sensor Networks: Algorithms and Architectures, A John Wiley \& Sons, 552 pages, ISBN: 978-0471-68472-5, 2005.

[2] ILYAS, M., MAHGOUB, I.: Handbook of Sensor Networks: Compact Wireless and Wired Sensing Systems, CRC Press, 672 pages, ISBN 0-8493-1968-4, 2004.

[3] JAYASHREE, L.S., ARUMUGAM, S., ANUSHA, M., HARINY, A. B.: On the Accuracy of Centroid based Multilateration Procedure for Location Discovery in Wireless Sensor Networks, Proc. Wireless and Optical Communications Networks, 2006

[4] SUNDARAM, N., RAMANATHAN, P.: Connectivity Based Location Estimation Scheme for Wireless ad hoc Networks, IEEE Globecom 2002, volume 1, pages 143-147, 2002.

[5] DOHERTY, L., PISTER, K. S. J., GHAOUI, L. E.: Convex Position Estimation in Wireless Sensor Networks, IEEE Infocom, volume 3, pages $1655-1663,2001$.

[6] NAGPAL, R., SHROBE, H., BACHRACH, J.: Organizing a Global Coordinate System from Local Information on an ad hoc Sensor Network, 2nd Intl. Workshop on Inform. Proc. In Sensor Networks, 2003.

[7] NICULESCU, D., NATH, B.: Ad hoc Positioning System, IEEE Globecom 2001, volume 5, pages 2926-2931, 2001.

[8] MAUVE, M, WIDMER, J., HARTENSTEIN, H.: A Survey on Position-Based Routing in Mobile Ad-Hoc Networks, IEEE Network, volume 15, issue 6, pages 30-39, 2001.

[9] KAEMARUNGSI, K., KRISHNAMURTHY, P.: Modeling of Indoor Positioning Systems Based on Location Fingerprinting, IEEE INFOCOM 2004.

[10] PRASITHSANGAREE, P., KRISHNAMURTHY, P., CHRYSANTHIS, P. K.: An Indoor Position Location with Wireless LANs, Proc. IEEE International Symposium on Personal, Indoor, and Mobile Radio Communications (PIMRC02), Lisbon, Portugal, 2002.

54 - KOMUNIKÁCIE / COMMUNICATIONS 4/2007 